\title{
An Application of the Electric Flicker Method to Tests of Physical Strength in Normals and Tuberculous Patients
}

\author{
By \\ Makoto Kobayashi \\ (小林 真) \\ From the Physiological Laboratory of Prof. K. Motokawa \\ Tohoku University, Sendai
}

(Received for publication, November 24, 1956)

\section{INTDORUCTION}

It has long been known that a subjective sensation of light, electrical phosphene, can be aroused by an electric stimulus passing through the eye. With this flickering phosphene as an index, Motokawa and his coworkers ${ }^{1}$ ) determined the threshold strengths for alternating currents of varying frequencies. Motokawa and Iwama ${ }^{1)}$ found a striking effect of oxygen lack upon this phenomenon, and further Motokwawa and Suzuki ${ }^{2)-5}$ reported that the electric flicker could be used as a very convenient indicator for measurements of fatigue.

Recently this method has been applied not only to the industrial hygiene, but also to the field of clinical medicine $e^{6 /-8)^{\circ}}$. In patients suffering from silicosis Nakamura et al..$^{9 / 10)}$ found that certain exercise greatly increased the electric flicker value or $\Delta \mathrm{S}$, and measured the time necessary for increasing the value at twice its initial value. The time so measured was found to serve for specification of the physical status of the patients.

Examination of the physical strength in tuberculous patients is important not only for making an appropriate therapy but also for determinating when they should resume their work.

This subject has been investigated by various workers with various methods; Kunishima, Abeno, Hirai and Sashida ${ }^{11-13)}$ and Yamada ${ }^{14}$ and Yuzawa $\epsilon t$ al. ${ }^{15}$ ) and others investigated the fatigue in tuberculous patients. In this connection the work by Nakamura et al.9/-10) mentioned above is especially worth noticing, because the load time they measured represented the physical status of their patients very satisfactorily.

It seems, however, that there are some points to be improved in their method, because too severe exercise was required to cause an increase $\Delta \mathrm{S}$ to twice the initial value. The circumstance that a recording apparatus 
of $\Delta \mathrm{S}$ is necessary for this method, is another shortcoming.

Therefore, it was attempted in the present investigation to reduce the trouble by measuring the time necessary to cause a least perceptible increase of $\Delta \mathrm{S}$, instead of an increase to twice the initial value. The method was applied to 43 healthy males, 37 healthy females and 210 patients suffering from pulmonary tuberculosis in the present investigation.

\section{EXPERIMENTAL \\ Method}

The method of electric flicker is described in detail in a previous paper ${ }^{1226) 7}$, so that only the point modified will be mentioned here.

At first, the mean of $10 \Delta \mathrm{S}$ values was obtained before a load exercise. The exercise consisted of a step test with a footstool which had two steps each $20 \mathrm{~cm}$. in height. The subject repeated movements up and down the stool every 5 seconds. The load time was 8-10 minutes for normal subjects, but 3-6 minutes for tuberculous patients. They were, however, told that they might give up exercise whenever they would. It is characteristic of the present method that a desired value of test can be obtained whatever the actual load time may be. The mean of $10 \Delta \mathrm{S}$ was obtained again immediately after the load exercise and the standard deviation (S.D.) was also calculated from this second series of measurements of $\Delta S$.

In Fig. 1 , in which mean values of $\Delta \mathrm{S}$ are plotted against load times, $a$

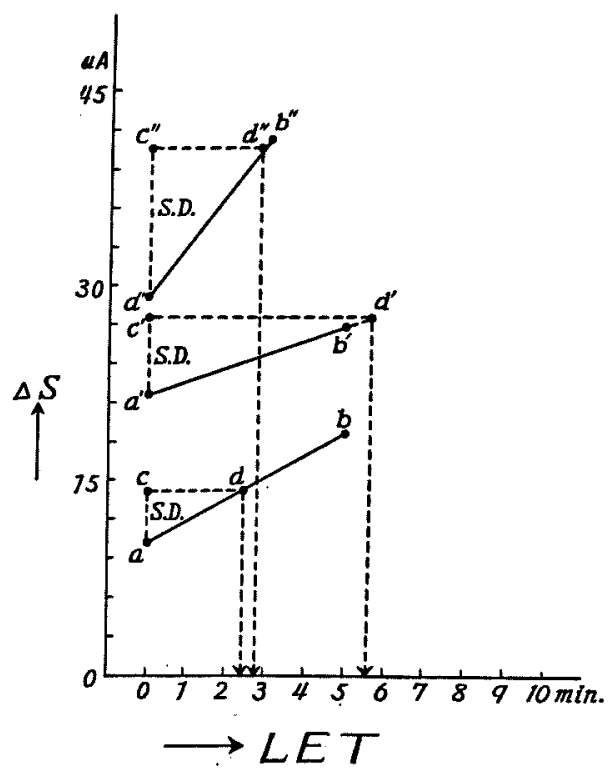

Fig. 1. Diagrams used for measuring LETs. Explanation in text, 
and $b$ represent the mean values of $\Delta \mathrm{S}$ before and after the exercise respectively. Similar pairs obtained from other cases are represented by $a^{\prime}$ and $b^{\prime}$ and by $a^{\prime \prime}$ and $b^{\prime \prime}$. Vertical lines $a c, a^{\prime} c^{\prime}$ and $a^{\prime \prime} c^{\prime \prime}$ represent S.D.s obtained from respective cases. $d, d^{\prime}$ and $d^{\prime \prime}$ represent points of intersection between horizontal line $c d$ and line $a b$, between $c^{\prime} d^{\prime}$ and $a^{\prime} b^{\prime}$, and between $c^{\prime \prime} d^{\prime \prime}$ and $a^{\prime \prime} b^{\prime \prime}$, respectively. Times corresponding to $d, d^{\prime}$ and $d^{\prime \prime}$ are measured, which will be denoted by "Least Effective Times" or LETs.

LETs are load times which would be required to increase the value of $\Delta S$ by its S.D. when it is assumed that $\Delta \mathrm{S}$ increases linearly with load time. The longer the LET, the greater the physical strength.

The normal subjects tested were 43 healthy males of 20 to 53 years in our laboratory, and 37 healthy females of 18 to 27 years of age who were mostly nurses, the averaged age being 26.5 years. The tuberculous patients investigated were 210 adults treated as inpatients or outpatients at 6 hospitals in the city of Sendai. They consisted of 161 males of 16 to 60 years of age and 49 females of 13 to 57 years of age, the averaged age being 33.2 years.

1) LETs of normal subjects

The results obtained are illustrated in Fig. 2, in which diagrams (A) and (B) represent respectively a histogram and a distribution curve of LETs for normals. In these diagrams LETs are expressed in logarithmic scale, because the distribution is more symmetrical in this expression than otherwise.

As can be seen in the figure, there is no appreciable difference between male and female.

In Table I daily variations of LETs of three normal subjects are shown. As can be seen in this table, daily variations are not so marked. Values of LETs taken in the morning and in the afternoon on the 10th day show no significant difference.

2) LETs of tuberculous patients

In Fig. 2(C) distribution curves obtained from tuberculous patients are shown in comparison with the normal control (the curve marked by open circles). The continuous curve marked by dots refers to 210 patients, and the broken one marked by crosses to 15 patients who had so recovered that were going to resume their work. There are significant differences among these three groups.

Fig. 2(D) represents distribution curves of LETs for three different groups, namely male outpatients (open circles), male inpatients (crosses) and female inpatients (dots). LETs of the male outpatients are generally longer than those of the inpatients.

(A) The relation between LETs and " the degree of rest cure" In national hospitals of Japan, tuberculous patients are classified into 


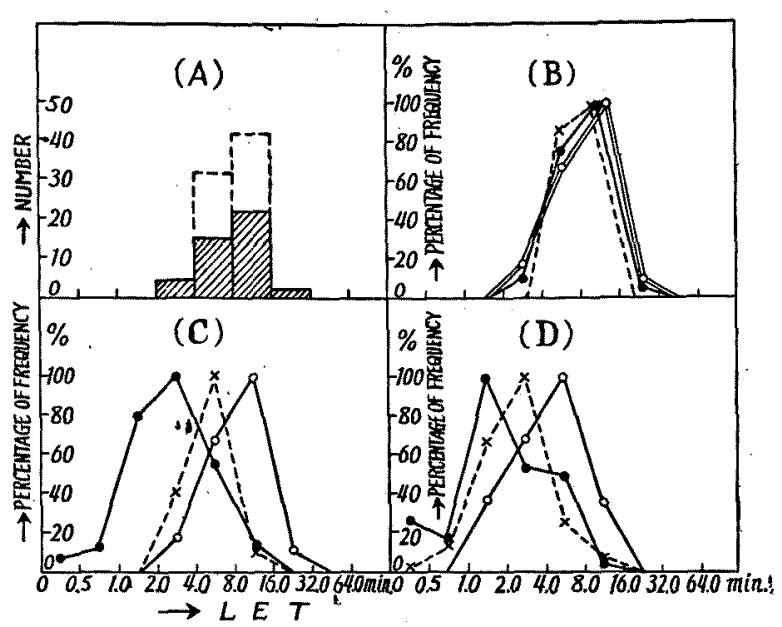

Fig. 2. Histograms and distribution curves of LETs obtained from normals and tuberculous patients. LETs are expressed in logarithmic scale.

(A) Histogram of LET obtained from 80 healthy adults. Rectangles drawn in full line and broken line refer to males and females, respectively.

(B) Distribution curves of LETs of normals. Curves marked by solid circles, by crosses and by empty circles refer to males, females and all together respectively.

(C) Distribution curves of LET obtained from normal adults, from patients just before resumption of office and from other patients marked by empty circles, by crosses and by solid circles respectively.

(D) Curves marked by solid circles, crosses and empty circles were obtained from male outpatients, male inpatients and female inpatients respectively.

8 classes according to the degree of rest necessary for each patient. The degree of rest is determined on the basis of various clinical examinations. The eight classes are labeled 1 to 8 from the most serious to the slightest one and this order is called "the degree of rest cure". The LET-test was applied to patients with various degrees of rest cure. The curve marked by dots in Fig. 3 (A) shows the relation between LETs and degrees of rest cure. The curve is almost linear, indicating a close oorrelation between them. It is to be noted that the degree of rest cure, which is determined by various clinical factors, is represented very satisfactorily by a single measure, such as LET. As indicated by curves marked by solid triangles and by open circles, neither $\Delta S$ values nor standard deviation measured immediately after the load exercise show any simple relation to the degree of rest cure.

(B) The relation between the LET and the time elapsed since a chest operation

In a number of patients who received a chest operation some months 
TABLE I

Daily Variations of LET in Minutes

\begin{tabular}{|c|c|c|c|c|}
\hline Day & Subjects & S.K. & M.K. & J.U. \\
\hline \multicolumn{2}{|c|}{1} & 10.8 & 9.0 & 7.5 \\
\hline \multicolumn{2}{|c|}{2} & 10.0 & 9.5 & 7.0 \\
\hline \multicolumn{2}{|c|}{3} & 8.7 & 7.5 & \\
\hline \multicolumn{2}{|c|}{4} & 14.4 & 9.5 & 5.0 \\
\hline \multicolumn{2}{|c|}{5} & 11.0 & 7.5 & 5.5 \\
\hline \multicolumn{2}{|c|}{6} & 12.0 & 8.0 & 6.0 \\
\hline \multicolumn{2}{|c|}{7} & 9.0 & 10.0 & \\
\hline \multicolumn{2}{|c|}{8} & 9.0 & 12.5 & 7.9 \\
\hline \multicolumn{2}{|c|}{9} & & 8.3 & 6.9 \\
\hline 10 & $\begin{array}{l}\text { a.m. } \\
\text { p.m. }\end{array}$ & $\begin{array}{r}9.0 \\
12.0\end{array}$ & $\begin{array}{r}11.3 \\
9.6\end{array}$ & $\begin{array}{l}6.5 \\
5.3\end{array}$ \\
\hline \multicolumn{2}{|c|}{ Mean } & 9.55 & 9.33 & 6.40 \\
\hline \multicolumn{2}{|c|}{ S.D. } & 1.43 & 1.31 & 1.01 \\
\hline
\end{tabular}

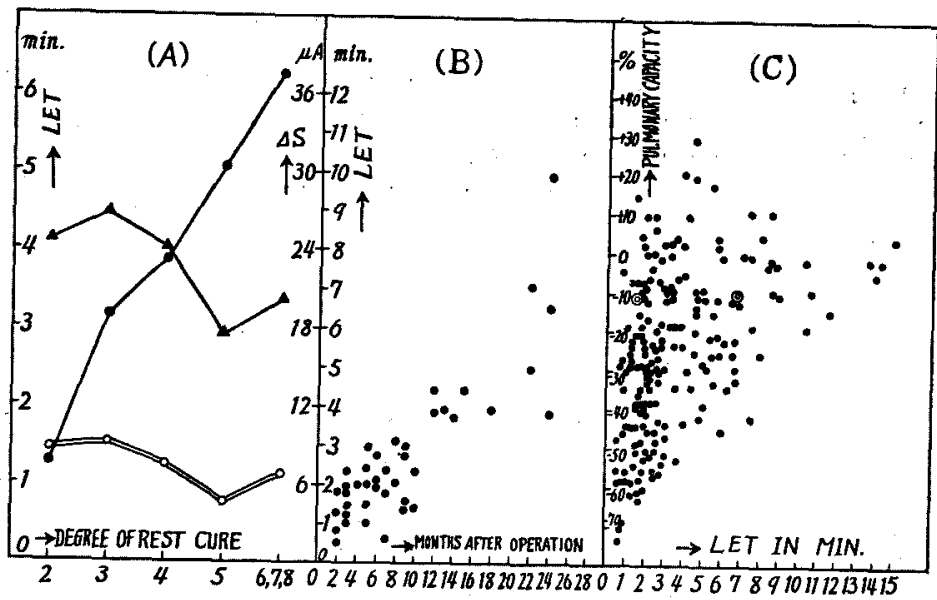

Fig. 3. Diagram (A) : Relations between degree of rest curve and LET (solid circles), $\Delta \mathrm{S}$ (solid triangles) and S.D. of $\Delta \mathrm{S}$ (empty circles).

Diagram (B): Relation between LET and months after chest operation.

Diagram (C) : Relation between LET and pulmonary capacity.

before, LETs were measured to see correlation between the LET and the number of months elapsed. The results are shown in Fig. 3 (B), which indicates that the longer the time elapsed, the longer the LET. This finding indicates that the LET can serve as a good indicator for recovery. 


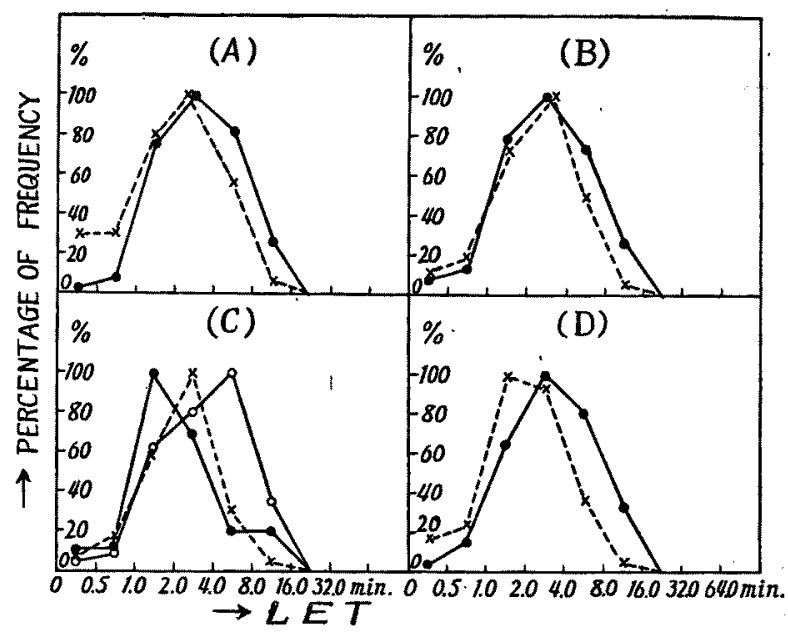

Fig. 4. Relation between LET and various kinds of clinical examination of 210 tuberculous patients.

(A) : Distribution curves of LET obtained from patients showing sedimentation rate below $10 \mathrm{~mm} / \mathrm{hr}$ (solid circles) and from those showing rate above $10 \mathrm{~mm} / \mathrm{hr}$ (crosses).

(B) : Distribution curves of LET in patients with bacilli positive (broken curve) and negative (continuous curve).

(C) : Distribution curves of LETs obtained from three groups of patients, far advanced (solid circles), moderately advanced (crosses), and minimally advanced (empty circles).

(D) : Distribution curves of LET in patients with pulmonary capacity below $-30 \%$ (full line) and over $-31 \%$ (broken line).

The same conclusion was reached from measurements of LETs in the entire course of recovery of certain 2 patients; the value of LET measured 2 months after a chest operation was found to be shorter than that found before the operation. The value increased gradually with time, but was found still lower than the initial level when measured 6 months after the operation.

(C) The relation between the LET and the pulmonary capacity

The percentage deviation from the standard vital capacity is defined as the pulmonary capacity. Negative values of it indicate decreases in vital capacity below normal. In Fig. 3 (C) pulmonary capacities of patients are plotted against corresponding values of LET. In general, there should be no simple relation between the pulmonary capacity and the LET, because the LET must be a function of various factors. In reality, the range of pulmonary capacity corresponding to any given value of LET is very wide, as can be seen in the figure, but it can not be overlooked that the minimal value of pulmonary capacity for each LET changes almost 
linearly with LET.

This fact may be accounted for as follows: When the pulmonary capacity is impaired so severely that it becomes a determining factor for the physical strength of a patient, then this factor becomes to determine his LET, because the LET is an indicator of the physical strength.

(D) The relation between the LET and other clinical signs

Tuberculous patients were classified into two groups concerning blood sedimentation rates; one group showing rates below $10 \mathrm{~mm}$ per hour, and the other having rates above $10 \mathrm{~mm}$ per hour.

In Fig. 4 (A) the distribution curves of LET for these two groups are compared. The continuous curve for the group having lower sedimentation rates is shifted to the longer side of LET. The difference between the two curves was found statistically significant at a 1 per cent level.

Simillarly the patients were divided into two groups according to whether tuberculous bacilli were positive or negative in sputum or gastric juice, and their distribution curves of LET were compared in Fig. 4 (B). These curves were shown to be significantly different at a 5 per cent level.

The roentgenograms of the patient's chests were classified into 3 categories, that is, far advanced, moderately advanced and minimally advanced according to the method of by American Trudeau Society. ${ }^{16)}$ In Fig. $4(\mathrm{C})$ the distribution curves of LET obtained from these 3 groups are shown. As will be seen in this figure, the curve for the far advanced group is situated on the left side, that for the minimally advanced group on the right side, and that for the moderately advanced group takes an intermediate position. Thus, values of LET correspond well to the severity of disease distinguished on the basis of roentgenograms.

Fig. 4 (D) refers to the distribution curves of LET obtained from 2 groups, showing pulmonary capacity less than $-30 \%$ and that greater than $-30 \%$. The difference between the two curves was found statistically significant at a 5 per cent level.

\section{Discussion}

Individual differences of $\Delta \mathrm{S}$ are generally so great that it is difficult to compare $\Delta S$ values obtained from different persons. Nakamura et al. ${ }^{910}$, tried to avoid this difficulty, taking a load time necessary for increasing $\Delta \mathrm{S}$ to twice its initial value as an index, instead of $\Delta \mathrm{S}$ itself. The load times of healthy adults were found, on an average, about 33 minutes, while those of patients suffering from silicosis ranged from 2 to 18 minutes. Therefore, it was suggested that the method might be used for clinical examination of the physical status of patients. The exercise used in the present investigation was not so severe as that used by Nakamura $e t$ al., and the load time was much shorter. This would be a great help in clinical ap- 
plication. The measurement of LET is also very easy and practical.

As has been demonstrated in Fig. 3 (A), a close relation between the LET and the degree of rest cure can be found, while no such correlation can be found between the value of $\Delta \mathrm{S}$ and the degree of rest cure. Some attempts were made in the present investigation to find any correlation between $\triangle \mathrm{S}$ and other clinical signs such as blood sedimentation rates, and roentgenograms, etc., but no significant correlation could be found.

Similar attempts were made with other measures such as the standard deviation of $\Delta \mathrm{S}, \Delta \mathrm{S}-\Delta \mathrm{S}_{0}$, and $\left(\Delta \mathrm{S}-\Delta \mathrm{S}_{0}\right) / \Delta \mathrm{S}_{0}$, where $\Delta \mathrm{S}_{0}$ means $\Delta \mathrm{S}$ value before a load exercise, but all these attempts failed. So far as my investigation goes, the LET seems to be the only practical measure obtained by the method of electric flicker. I should like to recommend the application of the present method to similar but other kinds of examination.

\section{SUMmary}

Electrical thresholds for appearance and disappearance of electric flicker were measured, and the difference of the two values was denoted by $\Delta \mathrm{S}$. The values of $\Delta \mathrm{S}$ were measured before and after a standard load exercise, a step test lasting several minutes.

A method was devised to find the time required for causing a least significant increase in $\Delta \mathrm{S}$ by the standard load. The time so measured was designated a LET (least effective time).

LETs were measured in 80 healthy adults and 210 patients suffering from pulmonary tuberculosis.

1. In the experiment on normals, any sex difference could not be found concerning LET.

2. Daily variations of LET were not so significant.

3. The distribution curve of LETs for male inpatients occupied a position intermediate between that for male outpatients and that for female inpatients.

4. A high correlation could be found between LET and the degree of rest cure, the number of months after a chest operation, or the pulmonary capacity.

5. LETs showed close correlation to various clinical signs such as sedimentation rates of blood corpuscles, roentgenograms of the chest, etc.

It was concluded from these findings that the LET serves as an excellent indicator for recovery of the physical strength in patients.

I am greatly indebted to Prof. K. Motokawa for his generous guidance and valuable suggestions.

\section{References}

1) Motokawa, K. \& Iwama, K., Tohoku J. Exp. Med., 1949, 50, 319. 
2) Motokawa, K. \& Suzuki, K., Jap. Med. J., 1948, 1, 200.

3) Motokawa, K. \& Suzuki, K., Nisshin Igaku (Jap.), 1948, 35, 523.

4) Suzuki, K., Tohoku J. Exp. Med., 1950, 52, 1.

5) Suzuki, K., ibid., 1950, 52, 9.

6) Mita, T., Abe, Z. \& Byonshik, T., ibid., 1951, 54, 45.

7) Motokawa, K., Umetsu, J., Kobayashi, M. \& Kameyama, M., ibid., 1956, 64, 151.

8) Umetsu, J. \& Suzuki, K., Tohoku J. Exp. Med., 1957, 65, 237.

9) Nakamura, Y., Nisshin Igaku (Jap.), 1953, 8, 1277.

10) Tsukahara, S., Umetsu, J. \& Kamei, F., Tohoku Ig. Z. (Jap.), 1954, 49, 316.

11) Kunishima, K., Abeno, T., Hirai, H. \& Sashida, G., Tairyoku-kagaku (Jap.), 1952, 2, 129.

12) Kunishima, K., Abeno, T. \& Sashida, G., ibid., 1952, 2, 133.

13) Kunishima, K., Abeno, T. \& Sashida, G., ibid., 1952, 2, 140.

14) Yamada, H., Kokenshi (Jap.), 1952, 3, 77.

15) Yuzawa, K., Ueda, Y., Yamagata, T. \& Abe, T., Kekkaku (Jap.), 1948, 23, 63.

16) National Tuberculosis Association, Diagnostic Standards and Classification of Tuberculosis, New York, 1955. 